\title{
3 Research Square

\section{Evaluation of the influence of genetic variants in Cereblon gene on the response to the treatment of ENL with thalidomide}

\section{Perpétua Socorro Silva Costa}

Universidade Federal do Maranhão

\section{Miriãn Ferrão}

Universidade Federal do Rio Grande do Sul

Thayne Woycinck Kowalski

Universidade Federal do Rio Grande do Sul

Lucas Rosa Fraga

Universidade Federal do Rio Grande do Sul

Mariléa Furtado Feira

Universidade Federal do Rio Grande do Sul

Luís Marcelo Aranha Camargo

University of São Paulo

Daniele lop Oliveira Caldoncelli

Centro Universitário São Lucas

Maria Irismar Silva Silveira

National Reference Center for Health Dermatology Dona Libânia - CDERM

Lavínia Schuler-Faccini

Universidade Federal do Rio Grande do Sul

Fernanda Vianna ( $\square$ fslvianna@gmail.com )

Universidade Federal do Rio Grande do Sul

\section{Research Article}

Keywords: Cereblon, thalidomide, erythema nodosum leprosum, pharmacogenomics

Posted Date: April 29th, 2021

DOI: https://doi.org/10.21203/rs.3.rs-415294/v1

License: (c) (1) This work is licensed under a Creative Commons Attribution 4.0 International License.

Read Full License 


\section{Abstract}

Erythema Nodosum Leprosum (ENL) is an acute and systemic inflammatory reaction of leprosy characterized by painful nodules and involvement of various organs. Thalidomide is an immunomodulatory and anti-inflammatory drug currently used to treat conditions such as ENL and multiple myeloma (MM). Cereblon protein was described as the primary target of thalidomide teratogenicity. Moreover, it was postulated that CRBN is necessary for the efficacy of thalidomide and its analogs in the treatment of MM. In this study, we evaluate the influence of CRBN genetic variants on the dose of thalidomide used in the treatment of ENL and on the manifestation of adverse effects during treatment for ENL. Polymorphisms rs1620675, rs1672770 and rs4183 located in regions flanking the part of the gene encoding the portion of the protein that binds to thalidomide were evaluated. An association with adverse ocular and gastrointestinal effects was observed. These results show that CRBN polymorphisms could alter the expression or activity of the protein and influence the development of adverse effects in response to thalidomide in ENL.

\section{Introduction}

Thalidomide is a glutamic acid derivative produced initially in the 1950s as an anticonvulsant and subsequently used as a sedative and antiemetic in early pregnancy ${ }^{1,2}$. However, its teratogenic effect soon became known when reports of birth defects in children born to mothers who used thalidomide during pregnancy appeared in 19613,4. Thus, it was withdrawn from the market, but next its importance was rediscovered according to the report of drug efficacy in the treatment of erythema nodosum leprosum (ENL) ${ }^{5}$.

ENL is a difficult to manage leprosy complication and a potentially disabling condition ${ }^{6,7}$. It is an inflammatory reaction characterized by painful nodules on the skin that can ulcerate, and by systemic involvement with fever and general malaise and effects on various organs ${ }^{7,8}$. The reaction affects patients with borderline lepromatous (BL) leprosy and lepromatous leprosy $(\mathrm{LL})$ that are associated with a higher bacillary load ${ }^{6,7}$.

Thalidomide is effective in treating ENL, rapidly reducing symptoms such as fever and night sweats and improving skin lesions ${ }^{1,5}$. By 1999, thalidomide was also proved to be effective in the treatment of multiple myeloma (MM) ${ }^{9,10}$ and later for other conditions. The effectiveness of thalidomide in ENL is initially due to its action on TNF-a, but other mechanisms may contribute to its anti-inflammatory effect 11,12 .

Recently, the Cereblon protein (CRBN) was described as the primary target of thalidomide teratogenicity ${ }^{13}$. This molecule is part of an E3-ubiquitin ligase complex (CRL4 ${ }^{\mathrm{CRBN}}$ ), acting as a substrate receptor that recognizes specific targets for ubiquitination, leading to further degradation by the ubiquitin-proteasome system ${ }^{14}$. More recently, it was shown that CRBN is necessary for the efficacy of thalidomide and its analogs lenalidomide and pomalidomide (named immunomodulatory drugs - IMiDs) in the treatment of 
$M M^{15-19}$. Several studies have investigated the Thalidomide-CRBN interaction with regard to the teratogenic, immunomodulatory and therapeutic effects of the drug. However, there are no studies on the effect of the Thalidomide-CRBN interaction on ENL.

CRBN gene encodes a protein 442 amino acids long, has 11 exons (NM_016302) and is highly conserved, hence, polymorphisms in its coding regions are rare. Therefore, some studies have analyzed non-coding regions of the gene that may be associated with the control of gene expression ${ }^{20-22}$. Three variants that flank the exons encoding the thalidomide-binding region of CRBN have been identified by bioinformatics tools as possible modulators of splicing sites with the potential to affect either the expression or activity of the protein ${ }^{23}$. They are located in regions adjacent to the region encoding the portion of the protein that binds thalidomide, an $A>C$ substitution ( $r$ 1620675) and a $G>A$ substitution ( $r$ 1672770), both in intron 10 , and an insertion/deletion (INS/DEL) of four nucleotides in (-/GTTA) in the 3'UTR region adjacent to exon 11 (rs4183). Here, we evaluated the influence of these three variants in CRBN on the variation of the dose of thalidomide and the occurrence of adverse effects of the use of this drug in patients with ENL.

\section{Results}

\section{Men may be more affected by lepromatous leprosy}

The demographic and clinical characteristics of the sample are presented in Table 1. In total, $103 \mathrm{ENL}$ patients were included, being $82(79.4 \%)$ male, and 78 (75.7\%) presenting lepromatous leprosy. 
Table 1

Clinical and demographic characteristics of ENL patients

\begin{tabular}{|ll|}
\hline Characteristic & $\mathbf{N}=103(\%)$ \\
\hline Male & $82(79.4)$ \\
\hline Multidrug therapy for leprosy & $44(43.9)$ \\
\hline Other medications & $84(81.3)$ \\
\hline Thalidomide dose [Median (P25/P75)] & $100(100 / 200)$ \\
\hline Days of consultation [Mean (min/max)] & $108.19(0 / 721)$ \\
\hline Patient Origin & \\
\hline Northeast Region & $90(86.9)$ \\
\hline North Region & $13(13.1)$ \\
\hline Leprosy classification: & $25(24.3)$ \\
\hline Borderline-lepromatous (BL) & $78(75.7)$ \\
\hline Lepromatous-leprosy (LL) & \\
\hline Adverse Effects: & $32(30.8)$ \\
\hline Neurological ${ }^{A}$ & $19(18.7)$ \\
\hline Grastrointestinal & $29(28)$ \\
\hline MusculoskeletalC & $19(18.7)$ \\
\hline Ocular ${ }^{\text {D }}$ & $16(15)$ \\
\hline Edema & $2(1.9)$ \\
\hline DermatologicalE & $12(11.2)$ \\
\hline Fever & A. Paresthesias,dizziness,tremor,neuritis, headache; B. diarrhea, vomiting, gastric fullness and \\
\hline pruritus, dry skin and hair loss. & \\
\hline
\end{tabular}

\section{Genotypic, allelic and haplotype frequencies of CRBN polymorphisms}

The genotypic distributions were in Hardy-Weinberg equilibrium and the allelic and genotypic frequencies of the polymorphisms are shown in Table 2. A high linkage disequilibrium (rs4183/rs1672770 ( $\left.D^{\prime}=0.925\right)$, rs4183/rs1620675 ( $\left.D^{\prime}=1.0\right)$, rs1672770/rs1620675 $\left(D^{\prime}=1.0\right)$ ) between the polymorphisms studied was identified (see Supplementary Table 1), and four haplotypes were identified in the sample (Table 3). 
Table 2

Genotypic and allelic frequencies of CRBN polymorphisms in ENL patients on treatment with thalidomide.

\begin{tabular}{|lll|}
\hline Polymorphism & Alleles/Genotypes & Frequency N (\%) \\
\hline rs1620675 & AA & $31(29.9)$ \\
& AC & $52(49.5)$ \\
& CC & $20(19.6)$ \\
\hline & A & $114(55.4)$ \\
\hline rs1672770 & AA & $92(44.6)$ \\
\hline & AG & $37(34.6)$ \\
\hline GG & $49(48.6)$ \\
\hline A & $17(15.9)$ \\
\hline G & $123(59.7)$ \\
\hline INS/INS & $83(40.3)$ \\
\hline INS/DEL & $21(19.6)$ \\
\hline DEL/DEL & $55(53.3)$ \\
\hline INS & $27(26.2)$ \\
\hline DEL & $97(47.1)$ \\
\hline
\end{tabular}

Table 3

Haplotype frequencies of CRBN

\begin{tabular}{|lcc|}
\hline Haplotype & $\mathbf{N}$ & (\%) \\
\hline INS/C/T & 3 & 2.8 \\
\hline INS/T/G & 71 & 70.3 \\
\hline DEL/C/T & 27 & 25.0 \\
\hline DEL/T/T & 2 & 1.9 \\
\hline Haplotypes in the following order: rs4183/rs1672770/rs1620675 \\
\hline
\end{tabular}


For all SNPs, there was no association of the thalidomide dose with time of treatment (Table 4). In addition, no association was found between haplotypes and thalidomide dose.

Table 4

Analysis of interaction between CRBN genotype and time related to estimated thalidomide dose using the generalized estimating equation model (GEE)* in erythema nodosum leprosum treatment

\begin{tabular}{|c|c|c|}
\hline Polymorphism & Interaction & $P$-value \\
\hline \multirow[t]{6}{*}{ rs1620675 } & rs1620675 & 0.110 \\
\hline & Concomitant Multidrug therapy for leprosy & 0.308 \\
\hline & Other medications & 0.198 \\
\hline & Region & 0.111 \\
\hline & Time & 0.001 \\
\hline & rs1620675*Time & 0.499 \\
\hline \multirow[t]{6}{*}{ rs1672770 } & rs1672770 & 0.302 \\
\hline & Concomitant Multidrug therapy for leprosy & 0.345 \\
\hline & Other medications & 0.031 \\
\hline & Region & 0.090 \\
\hline & Time & 0.001 \\
\hline & rs1672770*Time & 0.248 \\
\hline \multirow[t]{6}{*}{ rs4183 } & rs4183 & 0.195 \\
\hline & Concomitant Multidrug therapy for leprosy & 0.344 \\
\hline & Other medications & 0.191 \\
\hline & Region & 0.107 \\
\hline & Time & 0.001 \\
\hline & rs4183*Time & 0.983 \\
\hline \multicolumn{3}{|c|}{ Dependent variable: Thalidomide dose } \\
\hline
\end{tabular}


Fisher's test suggest CRBN polymorphisms could influence the development of adverse effects in response to thalidomide in ENL

In regards to the relationship of $C R B N$ variants and adverse effects of the use of thalidomide, it was found associations between the genotypes of rs1620675 ( $p=0.039), r s 1672770(p=0.024), r s 4183(p=$ 0.038 ) and gastrointestinal effects, which include diarrhea, vomiting, nausea, constipation and inappetence. (Table 5). The analysis of the association between haplotypes and adverse effects showed an association of ocular adverse effects $(p=0.030)$ with haplotype INS/C/T (rs4183/rs1672770/rs1620675) (Table 6). These effects were present in 2 patients (100\%) who carried this haplotype.

Table 5

Frequency of gastrointestinal adverse effects $A$

\begin{tabular}{|c|c|c|c|c|}
\hline Polymorphism & Genotype & $\begin{array}{l}\text { Absence-N } \\
\text { (\% within effect) }\end{array}$ & $\begin{array}{l}\text { Presence-N } \\
\text { (\% within effect) }\end{array}$ & P-value ${ }^{B}$ \\
\hline \multirow[t]{3}{*}{ rs1620675 } & AA & $28(26.4)$ & $4(3.8)$ & \multirow[t]{3}{*}{0.039} \\
\hline & $A C$ & $45(42.4)$ & $8(7.5)$ & \\
\hline & $\mathrm{CC}$ & 13 (12.7) & $8(7.5)$ & \\
\hline \multirow[t]{3}{*}{ rs1672770 } & AA & $25(23.6)$ & 12 (11.3) & \multirow[t]{3}{*}{0.024} \\
\hline & $A G$ & $45(42.4)$ & $7(6.6)$ & \\
\hline & GG & $16(15.1)$ & $1(0.9)$ & \\
\hline \multirow[t]{3}{*}{ rs4183 } & INS/INS & $13(12.3)$ & $8(7.6)$ & \multirow[t]{3}{*}{0.038} \\
\hline & INS/DEL & $48(45.3)$ & $9(8.5)$ & \\
\hline & DEL/DEL & $25(23.6)$ & $3(2.8)$ & \\
\hline
\end{tabular}


Table 6

Frequency of ocular adverse effectsA according to CRBN haplotypes

\begin{tabular}{|llll|}
\hline Haplotype $^{\text {B }}$ & Absence & Presence & P-value $^{\text {C }}$ \\
\hline INS/C/T C & $\mathbf{N}(\%)$ & $\mathbf{N}(\%)$ & 0.027 \\
\hline INS/T/G & $0(0)$ & $2(100.0)$ & $11(15.3)$ \\
\hline DEL/C/T & $61(84.7)$ & $6(22.2)$ \\
\hline DEL/T/T & $21(77.8)$ & $1(50.0)$ \\
\hline $\begin{array}{l}\text { A. Ocular adverse effects: decreased visual acuity and eye irritation; B. haplotypes in the following } \\
\text { order: rs4183/rs1672770/rs1620675; C. Fisher's exact test }\end{array}$ \\
\hline
\end{tabular}

\section{Discussion}

This study aimed to identify genetic variants in $C R B N$ gene that might influence in response of the treatment of ENL with thalidomide. We identified that all SNPs were associated with the manifestation of adverse gastrointestinal effects. In addition, an association of ocular adverse effects was found with the haplotype INS/C/T (rs4183/rs1672770/rs1620675).

CRBN acts as a substrate receptor as part of the E3 ubiquitin ligase complex (CRL4 ${ }^{\mathrm{CRBN}}$ ), which controls the expression of target proteins by their ubiquitination and degradation. According to Ito et al. (2010), CRBN is necessary for the teratogenic effect of thalidomide. In addition, this protein is also important for the anti-proliferative effect of thalidomide and other IMiDs in $\mathrm{MM}^{24}$. It is postulated that when thalidomide binds to CRBN, it modifies its function causing teratogenic effects by preventing the degradation proteins and/or by creating neosubtrates for ubiquitination and proteasomal degradation, which play a crucial role in embryonic development ${ }^{25,26}$. In the case of $\mathrm{MM}$, thalidomide binding to CRBN promotes recruitment of the neosubstrates Ikaros (IKZF1) and Aiolos (IKZF3) to the ubiquitin-ligase complex, resulting in increased ubiquitination and degradation of these transcription factors in $T$ cells and $\mathrm{MM}$ cells ${ }^{27-29}$. In addition, some studies have associated low CRBN mRNA expression with poorer clinical response to IMiDs, suggesting a potential role of CRBN as a predictive biomarker for treatment response ${ }^{17,26,28,30}$. Since the 1990s, it is known that one of the main effects of thalidomide is to decrease the TNF mRNA half-life, which explains some of its therapeutic effects. Using CRBN knockdown, it has been shown that the inhibitory effect of IMiDs on TNF-a production was also impaired in the silencing of $C R B N^{16,31}$.

$C R B N$ is composed of 11 exons extending over $30 \mathrm{~Kb}$. Thalidomide binds to $\mathrm{CRBN}$ in a region of 104 amino acids (339-442) located in the C-terminal portion, encoded by exons 9,10 and $11^{13}$. This gene is 
extremely conserved and a few polymorphism was found in the coding region ${ }^{23,32}$. Studies performed with $\mathrm{MM}$ showed that polymorphisms in non-coding regions of $C R B N$ were associated with response to thalidomide and others IMIDs therapy $20,21,29$.

The polymorphisms evaluated in the present study were identified in a previous study as possible splicing sites $^{23}$. The association of these SNPs and the dose of thalidomide could indicate that polymorphisms in these regions could interfere in the expression of the $C R B N$ gene or in the activity of $C R B N$, being able to modulate the response to treatment with thalidomide. However, in this study, we were unable to identify an association between these variants and the dose variation of thalidomide over time.

Nevertheless, in this study we found an association between all polymorphisms evaluated ( $\mathrm{rs} 1620675$ ( $p$ $=0.039), r s 1672770(p=0.024)$, rs4183 ( $=0.038))$ and the manifestation of gastrointestinal adverse effects. These adverse gastrointestinal effects consisted of diarrhea, vomiting, nausea and constipation. The most common symptom was constipation, a commonly reported side effect of thalidomide $22,33,34$. There was also an association of the haplotype INS/C/T (rs4183/rs1672770/rs1620675) with ocular adverse effects $(p=0.030)$, although only two patients have shown this haplotype. Such effects consisted of decreased visual acuity and eye irritation. Among these, only decreased visual acuity was present in the patients with this haplotype. The association of adverse effects with the polymorphisms studied may also be related to differences in CRBN expression. Mlak et al (2019) found an association between $C R B N$ variants (rs6768972, rs16727) and the risk of polyneuropathy and gastrointestinal disorders in patients with $M M$ treated with thalidomide-based regimens ${ }^{22}$. These variants are located in the $C R B N$ promoter region, thus they could influence the expression or activity of Cereblon ${ }^{22}$. It is important to highlight that the ocular adverse effects may also be associated with MDT for leprosy due to the use of clofazimine 35,36 . In fact, both concomitant use of multidrug therapy and use of other medications during ENL treatment may influence the appearance of adverse effects. In the analysis of the influence of polymorphisms on thalidomide dose, MDT and other medications were used as model covariates, but such correction was not possible in the case of influence analysis regarding adverse effects.

The epidemiological data of this study were in agreement with the data found in other studies ${ }^{7,37}$. Most of the individuals with ENL had lepromatous leprosy and were male. These frequencies corroborate that men may be more affected by lepromatous leprosy and that the bacillary index is a risk factor for the development of the reaction as demonstrated in other studies ${ }^{7,38,39}$. In addition, many patients were on MDT during treatment for ENL, confirming that the reaction manifests mainly in the first year of illness, during $\mathrm{MDT}^{40,41}$.

Some limitations should be considered during the interpretation of our study. The use of clofazimine in MDT for leprosy may interfere with both the dose reduction of drugs, because of its anti-inflammatory effect, and in the manifestation of adverse effects ${ }^{7,8}$. In addition, this is a retrospective study carried out with clinical data of patients, thalidomide dose, and manifestation of adverse effects obtained through 
the analysis of medical records. The lack of standardization in the description of this information or even the absence of a description of the adverse effects cannot be ruled out. Peripheral neuropathy, one of the main adverse effects of thalidomide ${ }^{1,8}$, was not evaluated in this study due to its retrospective nature, making it difficult to differentiate neuropathy due to the disease itself or due to the use of thalidomide. Accordingly, the evaluation of the effect of variants on the onset of peripheral neuropathy should be performed by a prospective study to reduce potential biases. Another limitation was that this study was carried out with individuals from different regions of the country and the heterogeneous genetic background of the Brazilian population might have been underestimated in the analysis and interpretation of the results. In addition, the limited sample size used in this study can become difficult the identification of minor effects from the genetic variants in different outcomes.

On basis of the results of this study, we identified some genetic variants of $C R B N$ were associated with adverse ocular and gastrointestinal effects. These results indicate that such variants could impact the protein and influence the outcome of treatment with thalidomide. This also indicates that CRBN may also be necessary for the action of thalidomide in ENL, as already described in $\mathrm{MM}^{24}$. Clearly, more studies evaluating the impact the variants in $C R B N$, as well as associations with ENL treatment must be performed in order to confirm this hypothesis. ENL is a chronic and difficult-to-control condition. Thalidomide is an effective drug, but has restrictions on its use due to peripheral neuropathy and its teratogenicity ${ }^{3,4}$. Therefore, it is important to identify useful biomarkers in predicting treatment response to limit its use to patients who will benefit most from treatment. To our knowledge, this is the first study to evaluate the association of genetic variants of $C R B N$ with thalidomide treatment in ENL patients. There are still many gaps to be filled in our knowledge of the mechanism of action of thalidomide and on how Cereblon participates in this process. Thus, this study shows that evaluation of $C R B N$ and its expression may help to understand the action of thalidomide in ENL and perhaps, in the future, be a useful biomarker in ENL.

\section{Materials And Methods}

\section{Ethical issues}

All participants were informed about the research objectives and signed an informed consent form. This study was approved by the Ethics Committee of the Hospital de Clínicas of Porto Alegre under number 10-04410 and CAAE 21184413.0.0000.5327. All research was performed in accordance with Brazilian regulations and informed consent was obtained from all participants. This research has been performed in accordance with the Declaration of Helsinki.

\section{Sample}

The sample consisted of 103 ENL patients who were selected from National Reference Center of Sanitary Dermatology Dona Libania in Fortaleza (Ceará State), Humanized Reference Center of Sanitary Dermatology in Imperatriz and Aquiles Lisboa Hospital in São Luís (Maranhão State) in Northeast Brazil, 
and from the Dermatology Ambulatory of the University of São Paulo in Monte Negro (Rondônia State) in north Brazil.

The patients used thalidomide at different doses and had a follow-up of up to six visits (average of 3.6 months).

\section{Clinical and demographic data analysis}

Up to six consultations annotated in the patient's medical record were analyzed with the collection of demographic data, including: sex, age and region of origin; history of leprosy (moment of diagnosis and treatment used); and history of ENL (diagnosis, treatment, adverse effects, history of relapse and dose of medications used).

\section{Genetic Analyses}

DNA was extracted from saliva samples using the Oragene DNA Extraction Kit (DNA Genotek), according to the manufacturer's instructions. A pair of primer was designed to amplify a fragment of 682 base pairs containing the region encompassing the three studied $C R B N$ polymorphisms: forward 5'-

TGTGGTCTTGGCAACCAGCAATTT-3' and reverse 5'-ACTGCCGTTCATGCTTGTTTCCT-3'. This region was amplified by polymerase chain reaction (PCR). The fragment obtained was visualized on a $2 \%$ agarose gel, purified and sequenced using the same primers.

Sequences were visualized and analyzed using CodonCodeAligner ${ }^{\circledR}$, version 3.0.1 (CodonCode Corporation, Dedham, MA, USA). The hg19 sequence deposited in GenBank was used as the reference sequence. When there was doubt about the variant, sequencing was repeated for confirmation.

\section{Statistical Analyses}

Chi-square test was used to evaluate Hardy-Weinberg equilibrium for all polymorphisms. Generalized estimating equations method (GEE) was used to evaluate the influence of $C R B N$ polymorphisms on thalidomide dose. This method is a repeated measures analysis focused on average changes in response over time and on the impact of covariates on these changes. GEE can model the average response of variables as a linear function of covariates of interest through a transformation or link function and can be used in studies where the data is asymmetric or the data distribution is difficult to verify due to the small-size sample ${ }^{42,43}$. The covariates inserted in the model were: place of origin of the patient, concomitant use of multidrug therapy (MDT) for leprosy, and use of other medications and other treatments for ENL.

The evaluation of the effect of $C R B N$ variants on the occurrence of adverse effects due to thalidomide treatment was conducted using Fisher's exact test. Peripheral polyneuropathy, although is an adverse effect common to the use of thalidomide, has not been evaluated because it is difficult to distinguish it from polyneuropathy caused by ENL and leprosy. All statistical analyses were performed with SPSS version 20 (SPSS, www.spss.com, IBM, USA). 
MLocus tool was used to calculate linkage disequilibrium (LD) for the polymorphisms ${ }^{44}$, and haplotypes were inferred using the Bayesian algorithm of the Phase 2.1.1 program ${ }^{45,46}$.

\section{Declarations}

\section{Data availability}

The data analyzed during the current study are not publicly available to maintain patient confidentiality. Moreover, this type of request has not been previously approved by participants nor the Human Research Committee. This data could, however, be available (anonymously) from the corresponding author on reasonable request.

\section{Acknowledgments}

We are grateful to INAGEMP (National Institute of Population Medical Genetics; Grant CNPq 573993/2008-4, 465549/2014-4, FAPERGS 17/2551.0000521-0 and CAPES), FIPE/HCPA (GPPG No. 100410) and Coordenação Brasileira de Aperfeiçoamento de Pessoal de Nível Superior (CAPES), for the support provided for this project. FSLV is recipient of a CNPq scholarship grant [grant number CNPq 312993/2017-0]. FSLV is recipient of a FAPERGS grant [grant number 19/2551-0001787-1]. We would also like to thank the collaborators who helped in obtaining the specimens at all the participating centers and the statistician Luciano Santos Pinto Guimarães for assistance with statistical analyses.

\section{Author contributions}

P.S.S.C. contributed devising the concept, designing and conducting the experiments, performing the statistical analyses, and writing the manuscript. M.F.M.F. contributed performing the statistical analyses and writing the manuscript. T.W.K. and L.R.F. contributed designing the experiment and correcting the manuscript. M.F.F. contributed conducting the experiments. Luís M.A.C., D.I.O.C., M.I.S.S. contributed obtaining the specimens and correcting the manuscript. L.S.F. contributed supervising the analyses and correcting the manuscript. F.S.L.V. contributed devising the concept, designing the experiments, supervising the analyses and correcting the manuscript. All authors discussed the results and contributed scientifically to the manuscript.

\section{Competing interests}

The authors declare no competing interests.

\section{References}

1. Melchert, M. \& List, A. The thalidomide saga. International Journal of Biochemistry and Cell Biology vol. 39 1489-1499 (2007). 
2. Mercurio, A. et al. A Mini-Review on Thalidomide: Chemistry, Mechanisms of Action, Therapeutic Potential and Anti-Angiogenic Properties in Multiple Myeloma. Curr. Med. Chem. 24, 2736-2744 (2017).

3. Mcbride, W. G. THALIDOMIDE AND CONGENITAL ABNORMALITIES. Lancet 278, 1358 (1961).

4. Lenz, W., Pfeiffer, R. ., Kosenow, W. \& Hayman, D. . THALIDOMIDE AND CONGENITAL ABNORMALITIES. Lancet 279, 45-46 (1962).

5. Sheskin, J. Thalidomide in the Treatment of Lepra Reactions. Clin. Pharmacol. Ther. 6, 303-6 (1965).

6. Chandler, D. J. et al. Household costs of leprosy reactions (ENL) in rural India. PLoS Negl. Trop. Dis. 9, e0003431 (2015).

7. Pocaterra, L. et al. Clinical course of erythema nodosum leprosum: An 11-year cohort study in Hyderabad, India. Am. J. Trop. Med. Hyg. (2006).

8. Costa, P. do S. S. et al. Erythema Nodosum Leprosum: Update and challenges on the treatment of a neglected condition. Acta Trop. 183, 134-141 (2018).

9. Singhal, S. et al. Antitumor Activity of Thalidomide in Refractory Multiple Myeloma. N. Engl. J. Med. 341, 1565-1571 (1999).

10. Ito, T. \& Handa, H. Cereblon and its downstream substrates as molecular targets of immunomodulatory drugs. Int. J. Hematol. 104, 293-299 (2016).

11. Moreira, B. A. L. et al. Thalidomide Exerts Its Inhibitory Action on Tumor Necrosis Factor oe by Enhancing $m$ R N A Degradation By Andre L. Moreira,* Elizabeth P. Sampaio, ${ }^{*}$ Antonina Zmuidzinas, ${ }^{*}$ Paula Frindt, ${ }^{*}$ Kendall A. Smith,* and Gilla Kaplan*. 177, 6-11 (1993).

12. Knobloch, J., Jungck, D. \& Koch, A. The Molecular Mechanisms of Thalidomide Teratogenicity and Implications for Modern Medicine. Curr. Mol. Med. 17, 108-117 (2017).

13. Ito, T. et al. Identification of a primary target of thalidomide teratogenicity. Science (80-. ). 327, 13451350 (2010).

14. Yang, J. et al. Cereblon suppresses lipopolysaccharide-induced inflammatory response through promoting the ubiquitination and degradation of c-Jun. J. Biol. Chem. (2018) doi:10.1074/jbc.RA118.002246.

15. Zhu, Y. X. et al. Cereblon expression is required for the antimyeloma activity of lenalidomide and pomalidomide. Blood 118, 4771-9 (2011).

16. Lopez-Girona, A. et al. Cereblon is a direct protein target for immunomodulatory and antiproliferative activities of lenalidomide and pomalidomide. Leukemia 26, 2326-2335 (2012).

17. Huang, S. Y. et al. Expression of cereblon protein assessed by immunohistochemicalstaining in myeloma cells is associated with superior response of thalidomide- and lenalidomide-based treatment, but not bortezomib-based treatment, in patients with multiple myeloma. Ann. Hematol. 93, 1371-1380 (2014).

18. Gao, S., Wang, S., Fan, R. \& Hu, J. Recent advances in the molecular mechanism of thalidomide teratogenicity. Biomed. Pharmacother. 127, 110114 (2020). 
19. Huang, P. A. et al. Cereblon gene variants and clinical outcome in multiple myeloma patients treated with lenalidomide. Sci. Rep. 9, 1-7 (2019).

20. Szudy-Szczyrek, A. et al. Polymorphisms in the promoter region of the CRBN gene as a predictive factor for the first-line CTD therapy in multiple myeloma patients. Oncotarget 9, 24054-24068 (2018).

21. Butrym, A. et al. Cereblon and IRF4 Variants Affect Risk and Response to Treatment in Multiple Myeloma. Arch. Immunol. Ther. Exp. (Warsz). 64, 151-156 (2016).

22. Mlak, R. et al. Polymorphisms in the promotor region of the CRBN gene as a predictive factor for peripheral neuropathy in the course of thalidomide-based chemotherapy in multiple myeloma patients. Br. J. Haematol. 186, 695-705 (2019).

23. Vianna, F. S. L. et al. Genomic and in silico analyses of CRBN gene and thalidomide embryopathy in humans. Reprod. Toxicol. 66, 99-106 (2016).

24. Gandhi, A. K. et al. Immunomodulatory agents lenalidomide and pomalidomide co-stimulate $T$ cells by inducing degradation of T cell repressors Ikaros and Aiolos via modulation of the E3 ubiquitin ligase complex CRL4CRBN. Br. J. Haematol. 164, 811-821 (2014).

25. Ito, T., Ando, H. \& Handa, H. Teratogenic effects of thalidomide: Molecular mechanisms. Cell. Mol. Life Sci. 68, 1569-1579 (2011).

26. Schuster, S. R. et al. The clinical significance of cereblon expression in multiple myeloma. Leuk. Res. 38, 23-28 (2014).

27. Chamberlain, P. P. et al. Structure of the human Cereblon-DDB1-lenalidomide complex reveals basis for responsiveness to thalidomide analogs. Nat. Struct. Mol. Biol. 21, 803-809 (2014).

28. Dimopoulos, K. et al. Expression of CRBN, IKZF1, and IKZF3 does not predict lenalidomide sensitivity and mutations in the cereblon pathway are infrequent in multiple myeloma. Leuk. Lymphoma 60, 180-188 (2019).

29. Iskierka-Jażdżewska, E. et al. Cereblon (CRBN) gene polymorphisms predict clinical response and progression-free survival in relapsed/refractory multiple myeloma patients treated with lenalidomide: a pharmacogenetic study from the IMMEnSE consortium. Leuk. Lymphoma 61, 699-706 (2020).

30. Heintel, D. et al. High expression of cereblon (CRBN) is associated with improved clinical response in patients with multiple myeloma treated with lenalidomide and dexamethasone. Br. J. Haematol. 161, 695-700 (2013).

31. Zhu, Y. X., Kortuem, K. M. \& Stewart, A. K. Molecular mechanism of action of immune-modulatory drugs thalidomide, lenalidomide and pomalidomide in multiple myeloma. Leukemia and Lymphoma vol. 54 683-687 (2013).

32. Butrym, A. et al. Polymorphisms within beta-catenin encoding gene affect multiple myeloma development and treatment. Leuk. Res. 39, 1462-1466 (2015).

33. Walker, S. L., Waters, M. F. R. \& Lockwood, D. N. J. The role of thalidomide in the management of erythema nodosum leprosum. Lepr. Rev. 78, 197-215 (2007). 
34. Wines, N. Y., Cooper, A. J. \& Wines, M. P. Thalidomide in dermatology. Australas. J. Dermatol. 43, 229-240 (2002).

35. Goulart, I. M. B., Leonel Arbex, G., Hubaide Carneiro, M., Scalia Rodrigues, M. \& Gadia, R. Efeitos adversos da poliquimioterapia em pacientes com hanseníase: Um levantamento de cinco anos em um Centro de Saúde da Universidade Federal de Uberlândia. Rev. Soc. Bras. Med. Trop. 35, 453-460 (2002).

36. Cholo, M. C., Steel, H. C., Fourie, P. B., Germishuizen, W. A. \& Anderson, R. Clofazimine: current status and future prospects. J. Antimicrob. Chemother. 67, 290-298 (2012).

37. Feuth, M. et al. Erythema nodosum leprosum in Nepal: a retrospective study of clinical features and response to treatment with prednisolone or thalidomide. Lepr. Rev. 79, 254-69 (2008).

38. Guerra, J. G. et al. Erythema nodosum leprosum case series report: clinical profile, immunological basis and treatment implemented in health services. Rev. Soc. Bras. Med. Trop. 37, 384-390 (2004).

39. Walker, S. L. et al. ENLIST 1: An International Multi-centre Cross-sectional Study of the Clinical Features of Erythema Nodosum Leprosum. PLoS Negl. Trop. Dis. 9, e0004065 (2015).

40. Voorend, C. G. N. \& Post, E. B. A Systematic Review on the Epidemiological Data of Erythema Nodosum Leprosum, a Type 2 Leprosy Reaction. PLoS Neglected Tropical Diseases vol. 7 (2013).

41. Dias, A. A. et al. DNA Sensing via TLR-9 Constitutes a Major Innate Immunity Pathway Activated during Erythema Nodosum Leprosum. J. Immunol. 197, 1905-1913 (2016).

42. Liang, K.-Y. \& Zeger, S. L. \{Longitudinal\} Data Analysis Using Generalized Linear Models. Biometrika 73, 13-22 (1986).

43. Sortica, V. A. et al. The effect of SNPs in CYP450 in chloroquine/primaquine Plasmodium vivax malaria treatment. Pharmacogenomics 17, 1903-1911 (2016).

44. Long, J. C., Williams, R. C. \& Urbanek, M. An E-M algorithm and testing strategy for multiple-locus haplotypes. Am. J. Hum. Genet. 56, 799-810 (1995).

45. Stephens, M., Smith, N. J. \& Donnelly, P. A New Statistical Method for Haplotype Reconstruction from Population Data. Am. J. Hum. Genet. 68, 978-989 (2001).

46. Stephens, M. \& Donnelly, P. A Comparison of Bayesian Methods for Haplotype Reconstruction from Population Genotype Data. Am. J. Hum. Genet. 73, 1162-1169 (2003).

\section{Supplementary Files}

This is a list of supplementary files associated with this preprint. Click to download.

- SupplementaryTable1.docx 